Culture et histoire dans l'espace roman

4 | 2010

Les représentations du corps dans la litterature latinoaméricaine

\title{
Son vacas, somos puercos ou l'utopie à corps perdu
}

\section{Marie-Caroline Leroux}

\section{(2) OpenEdition}

Journals

Édition électronique

URL : https://journals.openedition.org/cher/8712

DOI : $10.4000 /$ cher. 8712

ISSN : 2803-5992

Éditeur

Presses universitaires de Strasbourg

Édition imprimée

Date de publication : 30 juin 2010

Pagination : 215-226

ISBN : 978-2-35410-007-0

ISSN : 1968-035X

Référence électronique

Marie-Caroline Leroux, "Son vacas, somos puercos ou l'utopie à corps perdu », reCHERches [En ligne],

4 | 2010, mis en ligne le 15 décembre 2021, consulté le 25 janvier 2022. URL : http://

journals.openedition.org/cher/8712 ; DOI : https://doi.org/10.4000/cher.8712

\section{(c) (i) (2)(2)}

Ce(tte) œuvre est mise à disposition selon les termes de la Licence Creative Commons Attribution Pas d'Utilisation Commerciale - Partage dans les Mêmes Conditions 4.0 International. 


\title{
Son vacas, somos puercos ou l'utopie à corps perdu
}

\author{
Marie-Caroline Leroux \\ Université de Picardie Jules Verne
}

$\mathrm{D}$ éfinir le texte de la romancière mexicaine Carmen Boullosa en creux, en tâchant de déterminer ce qu'il n'est pas - roman de piraterie, roman historique ou simple badinage intertextuel - se révèle plus aisé que de le caractériser par l'affirmative. De prime abord, du fait du thème qui en est au cœur, on serait fondé à apparenter Son vacas, somos puercos aux romans d'aventures à la Stevenson. L'intrigue, sise en plein XVII e siècle, semble réunir tous les ingrédients du genre: une île, des pirates et un mystère. Le parcours initiatique de Smeeks, depuis son embarquement pour la Tortue jusqu'à son introduction finale aux arcanes de la Flibuste, répond à un schéma classique. Il n'est pourtant pas besoin de pousser bien avant la lecture pour constater les limites de ce modèle. Ce qui n'empêche pas d'autres ambivalences. Si les coordonnées de l'intrigue sont à mettre au compte de l'imagination de l'auteur, il n'en va par exemple pas de même pour le matériau de départ de la narration. Le récit est en effet élaboré sur la base d'authentiques mémoires, ceux du chirurgien Alexandre Exmelin, dont on sait qu'ils ont largement nourri, et les travaux d'historiens dans le domaine de l'étude de la piraterie, et l'imaginaire occidental. Néanmoins le pré-texte subsiste essentiellement ici sous forme de réminiscence. Au final, le double dialogue qu'instaure le roman avec un genre codifié et avec le texte dont il s'inspire échoue à en cerner la nature composite. Cet étrange objet littéraire se laisse difficilement embrasser, et le bizarre constat s'impose de ce que seuls l'inachèvement, le flottement et l'ambiguïté - toutes notions enracinées dans la négative - sont à même de circonscrire un vague champ dans lequel inclure Son vacas, somos 
puercos. Ainsi, quand même on ne s'attacherait qu'à offrir un cadre général au roman, on se trouverait immanquablement confronté à l'impression d'une carence.

Et il ne s'agit pas là du seul domaine où prévaut le motif de la vacance, souvent gémellé ici à celui de la perte. Il faut pour en saisir toute l'importance revenir à l'échelle de la diégèse, et interroger le problématique rapport à soi des personnages. L'un et l'autre terme s'imposent alors en clefs de lecture d'une œuvre dont la corporéité est un élément matriciel: en proie à une essentielle incomplétude, les protagonistes semblent sous le coup d'une menace permanente, soit qu'ils subissent des altérations de leur personne physique, soit qu'il leur faille satisfaire les nécessités d'un corps tyrannique. C'est là le premier point que l'on développera, avant de s'intéresser aux enjeux de la dépossession corporelle consentie par le narrateur. Elle lui permet, à l'issue de sa quête, d'accéder à une position surplombante sur le récit, position dont le degré ultime serait peut-être le «je» transcendé du chapitre métanarratif qui clôt la première partie du roman. Rompant avec la fiction pour insinuer la présence incarnée de l'auteur derrière un locuteur incertain et pour donner vie à l'objet-livre, le chapitre en question déroule le fil d'une association particulièrement féconde entre le texte et le corps. La connexion qu'y instaure la voix auctoriale avec le lecteur participe d'une somptueuse métaphore de l'acte de lecture, qui sera au cœur de la dernière partie de l'étude.

Au premier degré de la lecture, la relation de la douloureuse accession à l'âge adulte du protagoniste met d'emblée en scène un corps instrumentalisé. Dans cette partie de la trame fidèle au canevas des Flibustiers du Nouveau Monde d'Alexandre Exmelin, le personnage du même nom est d'abord reconfiguré sous les traits de Smeeks, jeune et naïf engagé. Débarqué à la Tortue, il se découvre tenu pour trois ans, par un contrat léonin, à une situation d'absolue servitude. Et ne tarde pas à apprendre qu'au sein de cette barbare utopie libertaire qu'est la Confrérie de la Côte, nul ne s'appartient qui n'ait conquis sa liberté, bien souvent aux dépens d'autrui. De son corps, donc, d'autres disposeront à leur guise. Comme outil de travail, mais aussi comme objet de plaisir. Ainsi Smeeks ${ }^{1}$ évoque-t-il explicitement les rapports intimes entretenus avec les deux hommes qui feront pour lui office de mentors, le guérisseur Negro Miel et le chirurgien Pineau. Consentie ou non, il convient ici de noter que la relation charnelle ne s'assortit guère chez

1 Le personnage est caractérisé par des désordres identitaires justifiant certaines variations onomastiques. Par commodité, il sera ici le plus souvent désigné sous ce nom. 
le protagoniste de l'expression d'une quelconque jouissance. Comme si là n'était pas sa vocation première: il semble bien en effet que le contact des corps garantisse ou s'accompagne essentiellement chez lui de la passation d'un savoir. Les connaissances auxquelles il accède par le truchement de ses relations avec Negro Miel et Pineau sont en partie d'ordre médical. En la matière, Smeeks s'ignore obstinément comme réceptacle de savoirs, alors que tout le proclame tel. Ainsi dans le passage où il se voit forcé de remplacer le défunt Negro Miel auprès des pensionnaires d'une maison close pour leur appliquer un remède connu de lui seul, la connaissance est-elle envisagée négativement, comme perte de l'innocence. Ignorant des réalités du beau sexe, et voyant les prostituées perdre du sang, il les croit à l'agonie et s'expose à leurs sarcasmes. Il faudra qu'elles lui fassent découvrir les talents qu'il possède (Boullosa: 51-52). Là pourtant n'est pas l'essentiel. Ses amis vont surtout lui transmettre les valeurs de la Confrérie, bien qu'il n'en prenne que tardivement conscience. C'est à la fin du roman que s'affirme véritablement son rôle de témoin (c'est-à-dire à la fois de spectateur et de passeur) d'une utopie moribonde, qu'il assume en devenant l'ultime signataire de la Loi de la Côte. Alors seulement on entrevoit une possible explication à l'emprise des sens sur le narrateur, affichée dès les premières lignes du texte:

¿Verlo? Todo lo he visto. Por algo tengo los ojos de J. Smeeks, a quienes algunos atribuyen el nombre de Oexmelin [...]. ¿Oírlo? Yo lo he escuchado todo, porque tengo también los oídos de Smeeks. Juntos, ojos y oídos, empezarán conmigo a narrar las historias de Smeeks en el mar Caribe y de aquellos con quienes compartí aventuras [...] (Boullosa: 13).

Vivant tatouage, le souvenir de l'utopie pirate marque son corps d'une empreinte toute sensorielle, qu'il revoie les flots de sang versés dans les assauts ou perçoive le bruit des chairs déchirées par la scie du chirurgien: «squish, squash, squish, oía yo al cuchillo entrando en el músculo» (Boullosa: 55). De sorte que, sous nos yeux, Smeeks devient effectivement le «libro escrito» de la Confrérie que Negro Miel se plaisait à faire de lui.

Mais revenons à l'idée d'assujettissement à laquelle se trouvent couplées les références à la corporalité. Dans cet espace où tout aveu de débilité se mue en promesse d'esclavage, il affecte naturellement le cortège des perdants de l'utopie (les engagés, les innocents torturés et massacrés, et enfin les femmes dans leur ensemble); pourtant les pirates ne s'appartiennent pas davantage, qui doivent se plier aux exigences d'un corps ivre de jouissance et de violence. Pour entendre la dimension aliénante de ces formes d'appropriation que sont chez eux, à des degrés divers, l'ingestion vorace 
d'alcool et de nourriture ou la torture ou le viol, il importe de les replacer dans le contexte plus vaste de l'utopie pirate. Anarchiste par essence, celle-ci exècre en théorie la propriété sous toutes ses formes. Schizophrène par la force, elle ne saurait sans conséquences pour elle se repaître de rapines et de la soumission d'autrui. Dans ce non-respect des valeurs qui la fondent réside sans doute, d'ailleurs, la raison ultime de son effondrement. Il est un champ où ce problème se pose avec une particulière acuité: la relation institutionnalisée entre l'homme et la femme. Promus par un gouverneur soucieux de fixer les flibustiers pour les mieux contrôler, le mariage et l'attribution d'un lopin de terre qu'il entraîne, en ce sens qu'ils légalisent une double propriété, déséquilibrent l'ensemble du microcosme pirate. Le nombre de femmes introduites dans l'île s'avère trop réduit pour que ne s'instaure une inégalité entre les uns et les autres. La femme devient source de discorde. Et, de fait, sa survenue sonne le glas de l'utopie. Ceci explique que le parchemin de la Loi associe la présence de la femme à la mort, et prône l'uranisme. Dans l'emprise sexuelle de chacun sur chacun qu'elle institue, la possession à double sens s'annule: «En el siguiente dibujo un hombre blanco usaba de un hombre negro y un negro usaba de un blanco" (Boullosa: 135). C'est cela précisément qui fonde une authentique égalité entre les Frères, seule à même de rendre pérenne l'idéal flibustier ${ }^{2}$.

Reste un dernier point à explorer s'agissant du corps comme réalité physique: il n'apparaît jamais comme le lieu de la plénitude de l'être à soi. Sa propension à trahir l'esprit par ses faiblesses et son aptitude à la douleur semble justifier une désolidarisation de ces deux entités. Aux femmes, la qualité de sujet plein est généralement interdite, et la dichotomie corpsesprit vient chez elles souligner une carence. Adèle, jeune prostituée qui tente en vain d'échapper à son sort, n'est plus que corps et donc, mutilée: «Sí, yo guardé silencio del cuerpo que se vendió desnudo y de su plan de fuga» (Boullosa: 74), écrit à son propos le narrateur dans une phrase où le corps devient support et supplée Adèle, sujet en absence. «Ella», femme qui aspire à vivre une utopie interdite à son sexe, est encombrée d'une enveloppe charnelle en inadéquation avec son être. Pour en finir avec le contrôle exercé par les hommes sur son propre corps, elle en est réduite à renoncer à lui et opte pour le travestissement masculin. Enfin ces riches créoles, violées puis réduites en charpie par les troupes de L’Olonnais, illustrent les dégâts

2 Encore que la notion de pérennité soit ici fort relative. En effet l'impossibilité physique de cette utopie toute masculine à assurer la reproduction de la vie la rend, de fait, stérile. L'exclusion de la femme ne préserve donc la Fraternité qu'en apparence. Mais ce n'est là qu'une des nombreuses contradictions de ce féroce lieu de perfection. 
occasionnés par la faillite d'un corps dont l'intégrité était pour elles gage d'honneur. Sa chute entraîne irrémédiablement le naufrage de la raison: elles sombrent dans la folie et s'immolent par le feu.

Les corps désirants ou souffrants - et dans tous les cas tourmentés - des personnages traduisent de profondes oscillations identitaires. Le malaise corporel semble bien clamer chez eux la nécessité d'accéder à un état d'harmonie entre l'âme et ce qui lui tient lieu de siège. Chez Negro Miel et Pineau, une identité s'est substituée à une autre avec le temps. Ces mélancoliques rescapés d'une époque révolue, amputés de leur qualité de pirates par la sédentarisation, étrangers à ce qu'ils ont été, n'apparaissent plus aux yeux du jeune Smeeks que comme de merveilleux conteurs. Le trépas seul résout cette fissure essentielle de leur être, dans l'extinction du corps. Leur mort relève d'une disparition d'ordre purement physique, car l'un et l'autre continueront d'habiter Smeeks - Negro Miel s'impose comme une voix narrative en surimpression tandis que Pineau s'invite avec insistance au travers du souvenir. Au final, il semble bien que l'anéantissement du corps ait une fonction cathartique: le traitement infligé par leurs assassins à Negro Miel et à son comparse étant précisément motivé par leur appartenance passée à la Confrérie, ils recouvrent, à l'instant fatal, leur identité perdue de messagers de l'utopie: le corps est sacrifié sur l'autel de l'harmonie retrouvée.

Les flottements identitaires se font plus perceptibles encore, pour ce qui touche au narrateur, dans les incertitudes de la dénomination. Si l'on veut bien convenir que l'identité d'un individu s'assied au premier chef sur le nom qu'il porte, il apparaît clairement que celui qui se désigne tour à tour comme Smeeks, Esquemelin et Le Trépanateur peine à trouver la sienne... En redonnant vie par le roman à celui qui s'était précisément efforcé de s'effacer comme «je» des Flibustiers du Nouveau Monde pour apporter plus de crédit à son témoignage, Boullosa complète son pré-texte sur un mode très personnel: le véritable Exmelin ne dit rien des années qui précédèrent son arrivée à la Tortue? Elle créera Smeeks, l'engagé. Il insiste sur son absence de prétentions en tant qu'écrivain? N'importe, elle le fera exister comme tel au travers du jeu métanarratif, avec Esquemelin. Il omet de souligner la part qu'il a pu prendre aux exactions commises par tous les mauvais sujets qui écumaient la mer des Caraïbes? C'est égal, elle en fera un gibier de potence digne de ses compagnons en lui offrant Le Trépanateur pour alter ego. Ces identités se distribuent dans la superposition. Le texte révèle l'effacement programmé de Smeeks, simple héros de ses aventures, 
au profit d'Esquemelin, écrivain, rapporteur et témoin du grand rêve de la Fraternité. À plusieurs reprises, la voix relègue Smeeks à l'arrière-plan pour mettre en avant Esquemelin: «[...] creo que desde un principio he sido Esquemelin, porque es él quien acostumbra narrar mi historia para que no se llame la atención sobre la persona de Smeeks, en sí de ninguna importancia» (Boullosa: 93). Il est à noter, en outre, que l'instabilité identitaire constitutive du personnage trouve son pendant dans l'indécision sur la personne narrative, tantôt hétéro, tantôt homodiégétique. Or c'est significativement à Smeeks que Boullosa réserve le regard en «il », concentré sur les premières pages du roman - c'est-à-dire, à l'échelle du discours, sur un temps antérieur à l'initiation du personnage. Smeeks - celui qui ne sait pas encore qui il est - n'a apparemment pas droit de cité dans la mémoire de la geste pirate, et le recours à la troisième personne pour le désigner l'en expulse symboliquement. Basculée en «il», cette part du «je» se voit concrètement appliquer le constat de ce que "yo ya no [soy] yo" (Boullosa: 105).

$\mathrm{Au}$ long de ce roman d'apprentissage, le protagoniste se dépouille de ces identités installées dans la stratification pour les transcender. Et la conscience de la corporéité a un rôle déterminant dans le lent cheminement vers soi-même du héros. Comme préalable à l'analyse de ce phénomène il faut établir clairement que, dans Son vacas, somos puercos, l'engagement somatique opère à deux niveaux distincts. Le paradoxe du corps tient ici à ce qu'il est tout à la fois ardente invitation à l'excentration, en tant que lieu du désir, et siège d'une multitude de perceptions engendrant une potentielle disponibilité au monde. Tout s'articule dans ce rapport duel, qui met en jeu les principes de l'actif et du passif, ou, plus symboliquement, du masculin et du féminin. Et ce qui ressort du parcours de Smeeks, c'est que le corps désirant a exercé sur lui une influence particulièrement délétère et a nourri son aveuglement. Car l'utopie ne se donne qu'à qui sait la voir, et la voir implique certains renoncements. L'ignorance dans laquelle est Smeeks de sa propre fissure le rend en quelque sorte absent à lui-même lors des campagnes de pillage. On voit alors Le Trépanateur - cette part de lui qui ne vit que pour le fer et la poudre - prendre le pas sur un «je» plus spacieux, dans le dédoublement:

Yo ya no era yo, ahora El Trepanador era el dueño de mis actos. En mis recordatorios, que debiera aquí reproducir, se contaban ochenta y cuatro piernas, y en mi persona no sabía cómo debía llevar la cuenta de las imágenes ultrajadas en las iglesias, ni de las españolas que había violado, ni las comidas que había hecho en mansiones, ni de las torturas presenciadas... ¿Hacía 
cuánto o cómo que yo era un enganchado, un esclavo, cuánto que dormía al aire libre, en el rellano de la casa donde crecí y de donde fui expulsado? No podía yo reconstruirme al regreso del asalto de Maracaibo. Yo ya no era nadie sino el puño que blandiera la espada chorreando sangre, el ojo apuntando, el dedo apretando el gatillo, aunque no fuera yo quien disparara y usara la espada, yo era los cuerpos que habían matado a veces con razón cuando se oponían a que les arrebatáramos sus trabajos y posesiones, a veces sin razón alguna, nada más por el gusto de verlos morir, de oír sus cuerpos caer, de salpicarnos con su sangre española, y yo era los cuerpos que había curado, en los que había puesto mi escalpelo, mi cincel, mi cuchillo... [...] ¿Me había perdido? Pero al preguntármelo yo sabía que lo que había perdido era mi cuerpo, que yo había sido sólo un esclavo, un engagé y que al dejar de serlo yo era el esclavo que perdió su cuerpo (Boullosa: 105-106).

Ces excroissances charnelles ( puño», «ojo», «dedo») qui agissent hors de toute volonté pour faire de lui l'antithèse du soigneur héritier de Pineau et Negro Miel constituent véritablement des corps étrangers. Et il est «el esclavo que perdió su cuerpo» en ce sens qu'il en a perdu le contrôle. Son corps le domine et le soumet: il ne s'est libéré de ses chaînes initiales que pour retomber dans une nouvelle servitude, autrement plus cruelle que celle qu'on lui avait imposée car il se l'inflige à lui-même, cédant au goût du sang, du sexe et de l'alcool. À ce stade son esprit, encore en inadéquation avec lui-même, cohabite vaille que vaille avec un corps despotique. Le véritable divorce n'est consommé qu'après la découverte de la Loi de la Côte et l'accession de Smeeks à la conscience de lui-même, qui s'accompagne d'une abdication de la corporéité. On saisit à cet instant la portée du poème qui figure en exergue du roman: en acceptant de n'être plus qu'une voix, celle du souvenir et de la transmission de l'idéal, le «je» est parvenu à ce point où les mots comblent la vacance du corps. Il peut devenir, comme dans le poème, "el esclavo que perdió su cuerpo / para que lo habiten las palabras" et s'attacher par l'écriture à offrir un écrin à l'utopie, à « trasmutar el tedio en ágatas / en oro el barro humano ». On le voit dès lors s'ouvrir à de puissantes esthésies, qui secrètent la mémoire. Ainsi les sens passent-ils de l'appétit inquiet à l'accueil des perceptions. Ainsi le principe masculin cède-t-il la place au féminin...

Smeeks s'abreuve donc au souvenir par les sens, préférentiellement par la vue et l'ouïe, qui hantent tout le roman et apparaissent ici naturellement complémentaires. On le constate lorsque le narrateur rapporte, non sans fascination, les leçons de chirurgie de Pineau. La typographie rend leur combinaison dans la simultanéité par une alternance de caractères italiques, pour rendre le discours; et de caractères normaux placés entre parenthèses, 
pour rendre le spectacle. Pineau marie le mot sur le geste et le geste même, de sorte que, sous nos yeux, cette leçon de médecine devient discours métaphysique et artistique (Boullosa: 55-56). On remarquera que les deux sens sur lesquels l'accent est mis ne requièrent pas de ce grand spectateur qu'est Smeeks un surinvestissement du monde qui l'environne, comme ce pourrait être le cas du toucher, plus facilement associable, peut-être, au désir qu'il a répudié. Par ailleurs la prééminence du sensoriel affecte directement le récit: elle génère une mémoire impressionniste qui fait fi de la rationalité, et dont l'aspect décousu des séquences qui composent le texte se fait l'écho. Le locuteur, au reste, en a pleine conscience:

Para un par de ojos y un par de oídos fijar las imágenes y los sonidos en el orden temporal en que ocurrieron no es tarea fácil, su memoria gusta burlar la tiranía del tiempo. Pero aunque salten a nosotros, desordenadas, imágenes como las de los pájaros atacando a los cangrejos en la arena de alguna isla Caribe para comérselos, corrompiendo el sabor de sus carnes tiernas con la hiel de los cangrejos que enturbian la vista y nublan la razón de quien los coma en exceso, y el sonido herrumbroso del tronar de sus duros picos destruyendo los carapazones, intentaré domarnos para empezar por el principio de la historia que deseo contar (Boullosa: 15-16).

Tout comme il boude la logique discursive, le «je» évanescent et affranchi des contingences corporelles qui nous conte l'histoire au présent ignore les barrières de l'espace et du temps, et même de celles de la mort. Si bien qu'il inaugure ouvertement une narration impossible, établie dans un ailleurs largement postérieur aux faits relatés: «[...] después de cientos de años aún me emociono (en el recuerdo) con el sueño de los Hermanos de la Costa [...]» (Boullosa: 80); «[...] ocurrió cuando ya nos habíamos disuelto, cuando Esquemelin había muerto hacía treinta años [...]» (Boullosa: 121); ou à l'inverse dans des temps antédiluviens, lorsque la voix spirite fait revivre le lointain Eden africain de Negro Miel.

Ce qui était chez lui fêlure se fait significativement tranquille acceptation dans la dernière étape de son parcours. Smeeks semble se perdre dans les passations de parole pour mieux sceller ses retrouvailles avec l'idéal. L'osmose ultime avec l'utopie se concrétise dans la translation du «je» vers un «nous». Ainsi, dans le passage précédemment cité, la première personne du pluriel, employée à deux reprises, implique-t-elle sans doute la présence de Negro Miel à ses côtés, voire, plus largement, celle de la communauté de la flibuste, bien que Smeeks s'emploie à garder le contrôle de son récit: «intentaré domarnos», dit-il. Ailleurs, ses remémorations sont plus ou moins insidieusement mises en abyme, et la voix devient celle de Negro Miel, 
ou encore celle de L'Olonnais, qui rapporte sa propre fin, comme doté de cette même faculté de Smeeks à survivre à son corps. Dans ce cas précis la scission corps-esprit est exposée avec d'autant plus de brutalité que ce corps est liquidé dans le dépeçage et la dévoration:

No me quemaron en una sola pieza: me asaron por partes, primero un miembro, después otro, otro... Yo aún estaba con vida cuando veía cómo los niños devoraban partes de mi cuerpo, comían trozos de mi propio cuerpo, hasta que la pérdida de sangre me hizo quedar sin conocimiento y exhalé el último aliento en el momento en que clavaban en mi torso una estaca para asarme con todo y cabeza (Boullosa: 131).

En tout état de cause, à mesure que le narrateur s'émancipe et s'abstrait de son corps, il tend à se libérer du personnage même qui l'a nourri. Ce qui se joue, c'est véritablement la déconstruction du sujet plein au profit d'un sujet narratif ductile, d'un «je» qui développe une singulière aptitude à devenir un autre...

Dans "(Número aparte», chapitre institué en laboratoire du métatextuel, Carmen Boullosa porte la voix à son plus haut degré d'épuration en mettant à nu un «je» qui réfléchit en abyme le processus de la lecture et de l'écriture tout en interrogeant l'objet-texte. Le segment, qui marque le passage à la deuxième partie du roman, se place ouvertement - typographiquement entre parenthèses. Celles-ci tracent une frontière entre le récit «horizontal» dominant et l'accident de la «verticalité » que constitue l'histoire de Negro Piedra, avatar de Negro Miel et personnage tout à fait étranger au récit principal. La corporalité que l'on croyait bannie se réaffirme en tout cas avec force dans "(Número aparte». Le locuteur, alors même qu'il en est arrivé au point culminant de sa maturation comme conscience mémorielle, se ré-incarne sous nos yeux:

Porque, sin tu cercanía, lector, sin la cálida compañía de tu cuerpo, yo no hubiera podido cruzar hacia arriba, en sentido vertical, la historia, porque cuando tu cuerpo se acerca a mí, yo me abandono, me dejo ir [...]. Así es cuando se acercan los cuerpos. La carne revela lo que ni la inteligencia ni los ojos pueden ver... A pesar de tu erotismo, firme y vigoroso, en el que me he dejado caer, como en el regazo de una hembra, meneándome hacia un lado y el otro como yo siento que tú me lo has pedido, sé que puedo caer, deshacerme, irme al cuerno, y conmigo todo cuanto he descrito aquí $[\ldots]$ (Boullosa: 65).

Il signe là le triomphe final du corps, et ce revirement requiert quelque éclaircissement. 
L'union charnelle constitue d'abord une invitation à contempler la mutation effective de la voix narrative en objet-livre. On l'a rappelé, Smeeks était considéré par les siens comme le «libro escrito» de la Fraternité; toutefois c'est bien autre chose qui est en jeu ici. Tout se passe comme si la pulsation de la mémoire se mettait par son intermédiaire à battre au cœur de l'ouvrage même que le lecteur a entre les mains, ce livre soudain chaud et vivant, proposé comme corps et offert à la sublimation dans l'acte d'amour. La mue opérée par le «je» le conduit à s'extraire à ce point de lui-même qu'il fait irruption dans un présent qui n'est même plus celui de l'écriture de ses mémoires mais bel et bien celui de la réception par le destinataire de son énoncé - "cuando tu cuerpo se acerca a mí, yo me abandono, me dejo ir », proclame-t-il. Carmen Boullosa ne nous redit-elle pas par là, avec une infinie poésie, que le geste sensuel et intime de la lecture est ce qui insuffle la vie au texte? Ce dernier redevient, au sens plein, le «tissu» dont il tire son étymologie: le verbe se fait chair dans le livre, ce médium grâce auquel l'auteur putatif du récit entre en contact direct avec le corps de son lecteur et triomphe des contraintes spatiotemporelles dont il se jouait déjà en tant que narrateur-personnage. Pour en terminer sur ce point, ajoutons que si l'étreinte éperdue que le narrateur-livre expose dans la métalepse atteste à ses propres yeux de la réalité de son être au monde, c'est peut-être en ce sens que le coït garantit la passation au narrataire de cette mémoire de l'utopie qui est si précieuse au protagoniste. Le lecteur de Smeeks-Esquemelin, érigé par le rapport sexuel en réceptacle, comme autrefois le personnage, hérite comme lui d'un savoir.

Livre, le sujet irrésolu de «(Número aparte» n'en est pas moins écrivain. Et comme tel il revendique la masculinité radieuse du geste scriptural, que complète un acte de lecture envisagé au féminin. Ce corps à corps puissamment érotisé - à l'inverse de la majeure partie des rapports sexuels qui émaillent le roman - scelle donc la réconciliation de deux principes jusqu'alors largement antagonistes. Par ailleurs le narrateur-écrivain se hisse ici si haut au-dessus de la fiction qu'on est porté à s'interroger sur sa véritable nature. N'a-t-il pas subrepticement cédé le pas à une authentique voix auctoriale, dévoilant l'écrivain Carmen Boullosa? Que ce "je» capricieux relève ou non de la figure autoreprésentative, il atteste par son propos de l'inquiète volonté d'exister du créateur, lequel ne semble devoir trouver de gage de sa propre matérialité que dans son œuvre et dans le miroir tendu par son public. En dernière analyse, les ébats du lecteur, du texte et de l'auteur 
mettent en scène la chimère d'une symbiose absolue, dans laquelle celui-ci se jette à corps perdu...

À la croisée de cette utopie textuelle et de celle de la grande Fraternité pirate, la problématique de la corporalité s'impose en clef de lecture de Son vacas, somos puercos. Elle charpente au demeurant une bonne part de l'œuvre narrative de Carmen Boullosa. Au fil de la réflexion elle aura permis de développer trois axes majeurs, autour d'un corps tantôt blessé et incomplet, tantôt sacrifié, tantôt réconcilié. J'en terminerai en pointant une autre approche possible de la corporéité dans le roman, qui passerait par le corps sexué. Car si dans «(Número aparte» elle révèle chez Boullosa la part de l'écrivain, on est tenté de conclure qu'à l'échelle du roman elle contribue à trahir l'auteur comme écrivain au féminin. Rien d'anodin dans le fait que les corps ici soumis et bridés soient surtout des corps de femmes, ou des corps d'hommes se comportant en femelles - en "vaches", dans la bouche des pirates. Le lieu n'était pas au développement de cette question, mais il convient de rappeler que Son vacas, somos puercos décrit un univers proscrit aux femmes et, par là même, réduit à la claudication, comme en atteste la triste fin de l'idéal flibustier. Peut-on par ailleurs attribuer au hasard le fait que le protagoniste s'épanouisse finalement dans la passation de parole et dans une corporéité apaisée, "parole» et "corps» renvoyant précisément à ce que Boullosa définissait il y a quelques années comme les armes de la féminité? Et Smeeks-Le Trépanateur fait-il autre chose que renoncer à ce qui, dans les termes de Boullosa, toujours, caractérise l'homme ?3 Pour autant, Carmen Boullosa ne proclame pas la défaite du principe masculin. Le roman signe bien plutôt une forme de dépassement des clivages génériques qui prend tout son sens dans «(Número aparte». Mêlant au féminin sa masculinité d'auteur-actant et se donnant à voir comme corps pleinement androgyne, le personnage inscrit là le texte dans l'ère de l'hybridité, cette autre utopie de notre temps.

3 «[...] según Boullosa, históricamente el hombre ha tenido el poder de la acción; mientras las mujeres han tenido sólo dos armas: «[...] el arma del cuerpo y el arma de la palabra»» (Cróquer Pedrón: 107) 


\section{Bibliographie}

Boullosa, Carmen, 1991, Son vacas, somos puercos, México, Era.

Cróquer Pedrón, Eleonora, 2000, El gesto de Antígona o la escritura como responsabilidad (Clarice Lispector, Diamela Eltit y Carmen Boullosa), Santiago, Cuarto Propio.

Exmelin, Alexandre-Olivier, 1996, Les Flibustiers du Nouveau Monde. Histoire des Flibustiers et Boucaniers qui se sont illustrés dans les Indes, Paris, Phébus [première édition, en néerlandais: 1678]. 